\title{
Patients with Premenstrual Syndrome Have Reduced Sensitivity to Midazolam Compared to Control Subjects
}

\author{
Inger Sundström, M.D., Sigrid Nyberg, and Torbjörn Bäckström, M.D., Ph.D.
}

\begin{abstract}
Premenstrual syndrome (PMS) depends on gonadal hormones produced by the corpus luteum. Given the facilitory actions on GABAergic inhibitory neurotransmission excerted by certain progesterone metabolites, further studies on the $G A B A_{A}$ receptor system in premenstrual syndrome are warranted. This study evaluated the benzodiazepine sensitivity in PMS patients and control subjects, using saccadic eye velocity (SEV) and visual analogue ratings of sedation as dependent measures. $P M S$ patients displayed a significantly reduced SEV responsiveness to benzodiazepines compared to control subjects in the follicular phase, whereas there was no difference between groups in the luteal phase. In the luteal
\end{abstract}

phase, the sedation response to benzodiazepines was significantly reduced in PMS patients compared to control subjects. There was also an influence of PMS symptom severity on these measures, as high-severity PMS patients displayed blunted SEV and sedation responses to benzodiazepines compared to low-severity patients. These results indicate that $P M S$ patients have a reduced functional sensitivity at the $G A B A_{A}$ /benzodiazepine receptor complex throughout the menstrual cycle. [Neuropsychopharmacology 17:370-381, 1997] (c) 1997 American College of Neuropsychopharmacology. Published by Elsevier Science Inc.

rect effects in the central nervous system (CNS) has focused attention on several neurotransmitter systems, presumably involved in the pathophysiology of PMS. Much evidence indicates that a serotonergic dysfunction is coupled to PMS (Bancroft et al. 1991; Sundblad et al. 1992), whereas studies on the $\mathrm{GABA}_{\mathrm{A}}$-transmitter system in PMS are more scarce. Progesterone metabolites such as the $3 \alpha$-hydroxy- $5 \alpha / \beta$ reduced metabolites (allopregnanolone and pregnanolone), allosterically bind to the $\mathrm{GABA}_{\mathrm{A}}$ receptor complex (GRC), thereby modulating inhibitory neurotransmission (Majewska et al. 1986). Brain and plasma levels of allopregnanolone temporally follow those of progesterone (Paul and Purdy 1992; Wang et al. 1996). Allopregnanolone has in several animal anxiety models been shown to exhibit anxiolytic effects (Bitran et al. 1993; Wieland et al. 1991) and has also been suggested to be involved in stress-response (Purdy et al. 1991).

Evidence of a possible role for the $\mathrm{GABA}_{\mathrm{A}}$-transmitter system in the pathophysiology of premenstrual syn- 
drome is mainly drawn from clinical trials on benzodiazepines. Alprazolam has been proven useful in the treatment of PMS (Freeman et al. 1995; Smith et al. 1987). Plasma levels of allopregnanolone correlate with well-being in PMS patients (Wang et al. 1996); however, no difference in plasma levels of pregnanolone and allopregnanlone between PMS patients and control subjects has been demonstrated (Wang et al. 1996; Schmidt et al. 1994). Cerebrospinal fluid (CSF) levels of GABA have been reported to remain unaltered across the menstrual cycle in PMS patients (Parry et al. 1991). The lack of consistent results regarding abnormalities in the $\mathrm{GABA}_{\mathrm{A}}$ transmitter system in PMS patients is primarily due to the difficulty of identifying measurable variables, reflective of GABA system function, in living human subjects. However, in recent years saccadic eye movement velocity (SEV) has been proposed as a sensitive measure on benzodiazepine/ $\mathrm{GABA}_{\mathrm{A}}$ receptor sensitivity. Benzodiazepines, acting via the $\mathrm{GABA}_{\mathrm{A}}$-receptor, are known to reduce the velocity of saccadic eye movements in a highly quantifiable, dose-dependent fashion (Hommer et al. 1986), and this reduction is blocked by the specific benzodiazepine antagonist flumazenil (Ball et al. 1991). The saccadic eye velocity is stable and reproducible within subjects, both between tests and within a testing period (Mercer et al. 1990) and once a saccade has been initiated, the velocity is not under conscious control. Saccadic velocity is generated by complex mechanisms involving the frontal eye fields, substantia nigra, superior colliculus, and pontine reticular formation (Becker 1989). When muscimol, a potent $\mathrm{GABA}_{\mathrm{A}}$ receptor agonist, is injected directly into the superior colliculus of a rhesus monkey, it produces a selective decrease in saccadic velocity. On the other hand, injection of bicuculline, a $\mathrm{GABA}_{\mathrm{A}}$ receptor antagonist, results in stereotyped and irrepressible saccades (Hikosaka and Wurtz 1985a). When injected into the substantia nigra, these substances produce opposite effects because neurons from this region exert a tonic GABAergic inhibition on saccade-related cells in the superior colliculus (Hikosaka and Wurtz 1985b). These regions contain the highest density of $\mathrm{GABA}_{\mathrm{A}}$-receptors in the brainstem (Okada 1992). Moreover, effects on SEV are highly correlated with sedation, which is another pharmacological action of the benzodiazepines (Hommer et al. 1986).

In a pilot study, we have recently demonstrated that PMS patients have a reduced SEV and sedation responsiveness to diazepam (Sundström et al. 1997). In an attempt to confirm our previous results we have, in the present study, increased the study group, measured effects over longer time, and used a different benzodiazepine compound (midazolam). Midazolam is a watersoluble benzodiazepine, which has a shorter half-life than diazepam and is less accompanied by administration problems. Furthermore, we also wanted to evalu- ate the severity of PMS and its influence on the SEV and sedation response to benzodiazepines.

Our hypothesis was that PMS patients would demonstrate a blunted SEV and sedation response to midazolam challenge compared to controls subjects. We also hypothesized that the severity of PMS symptoms would have relevance for these measures.

\section{MATERIAL AND METHODS}

\section{Subjects}

The patient group consisted of 12 physically healthy women between the ages of 25 and 45 with regular menstrual cycles, who sought treatment for severe dysphoric premenstrual changes of more than 6 months duration. The patients completed daily, prospective visual analogue ratings on 14 items descriptive of mood and physical symptoms and on six items descriptive of social and occupational impairment (Hammarbäck et al. 1989a). The daily ratings were filled in during two menstrual cycles to establish the diagnosis. Patients were considered to have PMS if they had a significant increase in at least five negative symptoms during 9 premenstrual days compared to 9 mid-follicular days, associated with a clinically significant social and occupational impairment (Hammarbäck et al. 1989a). All patients displayed at least 6 days of sparse symptomatology in the follicular phase and fulfilled the criteria of premenstrual dysphoric disorder according to the Diagnostic and Statistical Manual of Mental Disorders, 4th edition (APA 1994). Twelve physically healthy women between the ages of 25 and 45 with regular menstrual cycles and no significant premenstrual dysphoric symptoms in daily prospective ratings served as controls. All subjects completed a semi-structured interview and those taking oral contraceptives, other hormones, benzodiazepines or other psychotrophic medication were excluded. Those with an episode of mental disorder within the last 2 years and a history of drug abuse were also excluded from the study. No subjects consumed alcohol within $24 \mathrm{~h}$ of the test sessions. Caffeine use was restricted $3 \mathrm{~h}$ prior to, and throughout, the testings. Cycle phase was monitored by daily ratings continued throughout the study and confirmed by plasma progesterone sampled on each testing day. Subjects gave written informed consent and the study was approved by the Ethics Committee, University of Umeå, Sweden.

\section{Experimental Design}

Measurements were made on four occasions during the menstrual cycle; on 2 succeeding days in the follicular phase (days 6-12 of the menstrual cycle) and luteal phase (1-7 days prior to the onset of menstruation), respectively. The luteal phase intervals were chosen to 
correspond with maximum severity of mood symptoms rather than peak progesterone levels (Bäckström et al. 1983). Monitoring of luteal phase was confirmed by records on the next menstrual bleeding.

Testing was carried out in a hospital outpatient department. Subjects arrived in the morning or early afternoon. Scheduling was made according to the subject's wish, and the number of A.M. and P.M. testings were evenly distributed between groups as well as within individuals. An intravenous (IV) cannula was inserted in the forearm and blood samples were taken for estradiol and progesterone. The IV cannula was thereafter used for the intravenous injections. To establish baseline, three sets of SEV measurements and visual analogue ratings were made, with $5 \mathrm{~min}$ rest in between. Thereafter two IV injections of either placebo or midazolam were given with a 25-min interval. Midazolam was given in doses of $8 \mu \mathrm{g} / \mathrm{kg}$ and $16 \mu \mathrm{g} / \mathrm{kg}$. Equal volumes of saline was given as placebo. All injections were given over $60 \mathrm{~s}$. After the two injections of placebo/midazolam, SEV recordings were made at 5, 10, and 15 minutes, respectively. The second dose of placebo/midazolam was followed by additional SEV measurements made at 55, 70, 85, and $115 \mathrm{~min}$ (from the first midazolam injection). In connection to every set of SEV measurements, visual analogue symptom ratings were completed. Injections were given single-blindly. Double-blinding the injections was not considered to be effective due to the obvious sedative effect of the active drug. The placebo injections were always given on the day preceding the midazolam injections due to our wish to examine the patients for both placebo and $\mathrm{mi}-$ dazolam in the same menstrual cycle with a comparable hormonal environment. Plasma levels of progesterone and estradiol are known to change rapidly from day to day in the luteal phase. Furthermore, in PMS patients symptom severity varies from cycle to cycle, possibly due to varying steroid production by the corpus luteum (Hammarbäck et al. 1989b). Had placebo and benzodiazepine injections been given in a randomized order, a wash-out period of approximately 5-7 days between testing days would have been necessary. As this procedure would alter the timing between placebo and benzodiazepine injections in the luteal phase, the symptomatology and hormonal environment in subjects would differ considerably between treatments. Alternatively, placebo and benzodiazepine injections could have been given on the same day in separate cycles but for obvious scheduling reasons and because of the variation in plasma levels of gonadal hormones and PMS symptom severity between cycles, this was not a happy choice.

\section{Measurements of Eye Movements}

Saccadic eye velocity was measured using electrooculography (EOG) with the CSGAAS5 system, fully docu- mented elsewhere (Marshall et al. 1985; Marshall and Richens 1989). The test was performed in a quiet, semilighted room with the patient sitting in a comfortable chair. Head movement was prevented by supporting the subject's head with a pillow. EEG cup electrodes (Synetics AB, Stockholm, Sweden) with a small amount of electrode-gel (Elefix, Nihon Kohden) were used. After the skin had been scarified with Skinpure cream (Nihon Kohden), the electrodes were placed $1 \mathrm{~cm}$ laterally of the outer canthus of both eyes with one common electrode in the center of the forehead. Electrode impedances were measured and confirmed to be less than $5 \mathrm{kohm}$. The subject was instructed to watch an array of light-emitting diodes (LED), placed at eye-level, $67 \mathrm{~cm}$ from the glabella. The subject was asked to look at the illuminated LED and as that LED was turned off and the next one in the array was lit, to move her eyes to the next target. Subjects were instructed not to anticipate targets. The target movements took place at 1.5-s intervals. A fixed, non-random sequence of $4 \times 20$ targets producing target steps of 10,20,30, and 40 degrees, was displayed with a brief rest in between. The EOG was DC amplified and low-pass filtered $(-3 \mathrm{~dB}$ at $50 \mathrm{~Hz})$ before being digitized to 12-bit resolution at a sampling frequency of $250 \mathrm{~Hz}$. A personal computer controlled the target movements and digitized the waveform using an A-D converter. The 80 individual EOGs were analyzed according to the method of Marshall and Richens (1989). First, the digitized data from each target displacement was processed to locate saccades. Secondly, each saccade was analyzed to determine the size of the saccade in degrees and the peak saccadic velocity. Thereafter a velocity-saccade size curve, known as the main sequence, was plotted (Baloh et al. 1975). The relationship between actual target displacement and peak velocity is important, since it remains intact even when voluntary control of saccades is attempted. The main sequence was fitted by a quadratic equation to the peak velocity data using the calculated saccade angle as the independent variable. The influence of outliers in the data was minimized by carrying out the fitting procedure twice and weighing the second fit with the inverse of the square of the residuals from the first fit. The values for peak velocity for $10,20,30$, and 40 degrees saccades were then calculated by interpolation. Saccades with 30 degrees amplitude were chosen for further analyses because peak saccadic velocity reaches a maximum at approximately 30-35 degrees of angular movement (Baloh et al. 1975).

\section{Visual Analogue Ratings}

During the two diagnostic cycles and throughout the testing cycle/cycles, the women filled out a daily rating scale. In total, four negative mood parameters, four positive mood parameters, three behavioral parameters, 
and three somatic symptoms were rated as was the severity of menstrual bleeding. For each item, the subject marked on a $10-\mathrm{cm}$ line to indicate how she had experienced a particular symptom during that day, with 0 as complete absence of symptoms, and 10 as the maximal severity of the symptom. Throughout the testing days similar visual analogue scales were used after every set of SEV measurements. The subjects were asked to rate six symptoms; cheerfulness, relaxation, anxiety, sedation, depression, and irritability. Visual analogue scales have been found to be an effective tool in measuring changes over time in response to treatment for mood symptoms and their reliability and validity have been well documented (McCormack et al. 1988).

\section{Statistics}

The three baseline SEV measurements and visual analogue ratings were calculated into meaned baseline scores. Saccadic eye velocity, self-ratings of sedation, and other mood symptoms were calculated as delta scores (difference from the meaned baseline at each time point). Eleven time-points were available; one meaned baseline and 10 post-injection measurements. The first six measurements represented acute benzodiazepine effects (three time points after each of the two injections) and the following four measurements the off-set back to baseline. The focus in this study was on the acute effects of benzodiazepines.

To subdivide the PMS group into a high-severity and low-severity group, the number of expressed symptoms per day during the premenstrual period was used as dividing ground (Wang et al. 1996). The visual analogue ratings of the testing cycle was used for this purpose. The total number of days with expressed symptoms (symptom score $>1$ ) in the 10-day premenstrual period was counted for the four negative mood symptoms. The higher the total number of expressed symptoms per day, the more severe PMS the patient was experiencing. A day with a negative symptom score of 0 1 was defined as symptom-free. This definition permitted the comparison of expressed symptoms between individuals, whereas visual analogue ratings are subjectively defined and less suitable for between-subjects analyses. The PMS patients were subdivided into two equally large groups, a high-symptom group $(n=6)$ and a low-symptom group $(n=6)$.

Four-way analysis of variance (ANOVA) was used on the SEV results, sedation, and mood change scores. The appropriate model would have been a repeated measures ANOVA, but due to the large number of intra-individual repetitive measures, a univariate model was chosen. The independent factors were time (change with time after each drug dose), phase (follicular vs. luteal phase), drug (placebo vs. midazolam), and group (control subjects vs. PMS patients or high-severity pa- tients vs. low-severity patients). The focus of these analyses was whether there was: (1) a dose-related effect of midazolam compared to placebo (drug by time), (2) whether there was a difference between groups in the response to benzodiazepines (group by drug effect), (3) whether this difference was dose-related (group by drug by time), (4) whether the difference between groups depended on cycle phase (group by drug by phase), and (5) whether a difference in sensitivity to benzodiazepines between cycle phases (phase by drug effect) existed. As far as possible, the results presented were drawn from this analysis. However, to clarify certain aspects, two-way ANOVA on the post-benzodiazepine results were performed. Accordingly, group differences in each cycle phase were tested by two-way ANOVA on the post-benzodiazepine results with group and time as independent factors. Phase differences in each group was tested by two-way ANOVA on the post-benzodiazepine results with phase and time as independent factors. Whenever there was an interaction with time, post-hoc analyzes by Tukey's Honestly Significant Difference Test were made. One-way ANOVA was made in each group and phase on the placebo and benzodiazepine response over time. Diagnostic mood ratings were evaluated by three-way ANOVA with group, cycle day, and phase as independent variables. For intraindividual comparison between menstrual cycle phases of hormone levels and visual analogue ratings in each respective group, the Wilcoxon MatchedPairs Signed-Ranks Test was performed. Differences between groups in hormone levels were evaluated by the Mann-Whitney U-test. As normal distribution could not be assured, due to small sample size, nonparametric tests were used. Correlations between SEV and mood symptoms were made by partial correlation where the effect of time was controlled for. Missing values were excluded from the analyses. All values are displayed as mean \pm SEM. The SPSS statistical package was used for all analyses.

\section{RESULTS}

Patients and control subjects were similar in age (patients $36.5 \pm 1.3$, control subjects $36.4 \pm 1.2$ ). Based on the progesterone levels $(>15 \mathrm{nmol} / \mathrm{l})$, cycle length and onset of next menstrual bleeding, all subjects were considered to have had ovulatory cycles. During the luteal phase, the PMS group showed significant increases in irritability $\mathrm{F}(1,276)=219 ; p=.001$, depression $\mathrm{F}(1,276)=$ $219 ; p=.001$ and anxiety scores $\mathrm{F}(1,276)=150 ; p=.001$ compared to control subjects. The control subjects, on the other hand, exhibited constantly low levels of symptoms throughout the menstrual cycle. One PMS patient reported a prior episode of depression, and one PMS patient had experienced a post-partum depres- 
Table 1. Mean \pm SEM of Estradiol and Progesterone Plasma Levels in PMS Patients and Control Subjects with Respect to Different Menstrual Cycle Phases

\begin{tabular}{|c|c|c|c|c|}
\hline & \multicolumn{2}{|c|}{ Control Subjects } & \multicolumn{2}{|c|}{ PMS Patients } \\
\hline & $\begin{array}{l}\text { Estradiol } \\
(\mathrm{pmol} / \mathrm{l})\end{array}$ & $\begin{array}{l}\text { Progesterone } \\
\text { (nmol/l) }\end{array}$ & $\begin{array}{l}\text { Estradiol } \\
\text { (pmol/1) }\end{array}$ & $\begin{array}{l}\text { Progesterone } \\
\text { (nmol) }\end{array}$ \\
\hline Follicular phase & $202 \pm 27$ & $2.0 \pm 0.2$ & $261 \pm 20$ & $2.3 \pm 0.3$ \\
\hline Luteal phase & $270 \pm 29$ & $25.3 \pm 3.6^{\mathrm{b}}$ & $293 \pm 34$ & $14.8 \pm 2.0^{\mathrm{a}, \mathrm{b}}$ \\
\hline
\end{tabular}

sion. Control subjects were devoid of psychiatric illness, and there was no report on prior use of benzodiazepines in either group.

One control subject became pregnant in the menstrual cycle following her luteal phase testings, and therefore only her luteal phase values were included.

One control subject became so sedated after the first midazolam injection in the luteal phase that the second injection was not given for safety reasons. All other subjects received both their injections, but at various times some subjects were unable to fixate and follow the LED. In the follicular phase, after midazolam injections, control subjects failed to perform saccades at six times and one PMS patient at one time. In the luteal phase, after midazolam injections, control subjects failed at three times and PMS patients at two times. There were also a small number of missing values during the placebo injections, equally spread over groups and phases.

Control subjects and patients did not differ with respect to the timing of testings for follicular phase (cycle day $9.2 \pm 0.34$ vs. cycle day $9.5 \pm 0.43$ ), whereas controls were tested earlier in the luteal phase, although not significantly (cycle day $-4.0 \pm 0.54$ vs. cycle day $-3.0 \pm 0.58$ ).

Estradiol and progesterone levels for each respective group and phase are shown in Table 1. In the luteal phase, PMS patients had significantly lower progesterone levels than control subjects, $p=.017$. This finding was presumably due to the difference in timing between groups in the luteal phase. As expected, progest- erone levels increased significantly between phases in both groups, $p<.001$, respectively.

\section{Saccadic Eye Movements}

PMS patients had lower baseline SEV than control subjects $F(1,86)=6.06 ; p=.016$. There was no group by phase interaction, indicating that the difference between groups in baseline SEV persisted throughout the menstrual cycle (see Table 2). Results from the overall SEV response to placebo and midazolam, including data from both groups and cycle phases, are shown in Figure 1. Compared to placebo, midazolam injections induced a significant dose-related SEV response $\mathrm{F}(5,499)=2,93$; $p=.013$. Post-hoc analyzes are given in Figure 1 .

Results from the acute SEV response (the first six time-points) to the midazolam injections for each group and phase are shown in Figure 2. Midazolam injections induced a significant decrease in SEV in both groups and cycle phases (control subjects follicular phase $\mathrm{F}(5,54)=3.93 ; p=.0041$, luteal phase $\mathrm{F}(5,60)=2.65 ; p=$ .032 , PMS patients follicular phase $\mathrm{F}(5,65)=4.37 ; p=$ .0017 , luteal phase $\mathrm{F}(5,64)=6.60 ; p=.001)$. Following placebo injections, there was no significant decrease in SEV in either group or phase (not shown).

The four-way ANOVA revealed a significant drug by group by phase interaction in SEV response $F(1,499)=$ $4.39 ; p=.037$ (Figure 2). By analyzing the menstrual cycle phases separately by two-way ANOVA on the postbenzodiazepine SEV results, this three-way interaction

Table 2. Mean \pm SEM of Saccadic Eye Velocity and Sedation Scores at Baseline on the Midazolam Testing Days

\begin{tabular}{lccccc}
\hline & \multicolumn{2}{c}{ Control Subjects } & & \multicolumn{2}{c}{ PMS Patients } \\
\cline { 2 - 3 } & Follicular Phase & Luteal Phase & & Follicular Phase & Luteal Phase \\
\hline $\begin{array}{l}\text { Saccadic eye } \\
\text { velocity, deg/s }\end{array}$ & $459 \pm 11$ & $470 \pm 11$ & & $420 \pm 20$ & $432 \pm 19$ \\
Sedation & $0.85 \pm 0.29$ & $1.39 \pm 0.65$ & & $2.86 \pm 0.82$ & $4.47 \pm 0.77$ \\
\hline
\end{tabular}

PMS patients had lower SEV than control subjects in both phases of the menstrual cycle $\mathrm{F}(1,86)=6.06 ; p=$ .016. There was no difference in sedation ratings between cycle phases in either group. 
was further clarified. Accordingly, the PMS patients showed a reduced responsiveness to midazolam compared to control subjects in the follicular phase $F(1,119)=$ $17.73 ; p=.001$, whereas there was no difference between groups in the luteal phase $\mathrm{F}(1,124)=1.71 ; p=$ .19. There was no interaction with time in either cycle phase, indicating that the group difference present in the follicular phase was not dose-related, but generally present over all measurements. Due to the significant difference in baseline SEV a four-way ANCOVA correcting for pretreatment levels was made, in which the drug by group by phase interaction was preserved $\mathrm{F}(1,584)=4.08 ; p=.044$. Maximum percent reduction in SEV following midazolam injections in the follicular phase was $30.3 \pm 5.3 \%$ among control subjects and $21.7 \pm 1.9 \%$ among PMS patients. In the luteal phase,

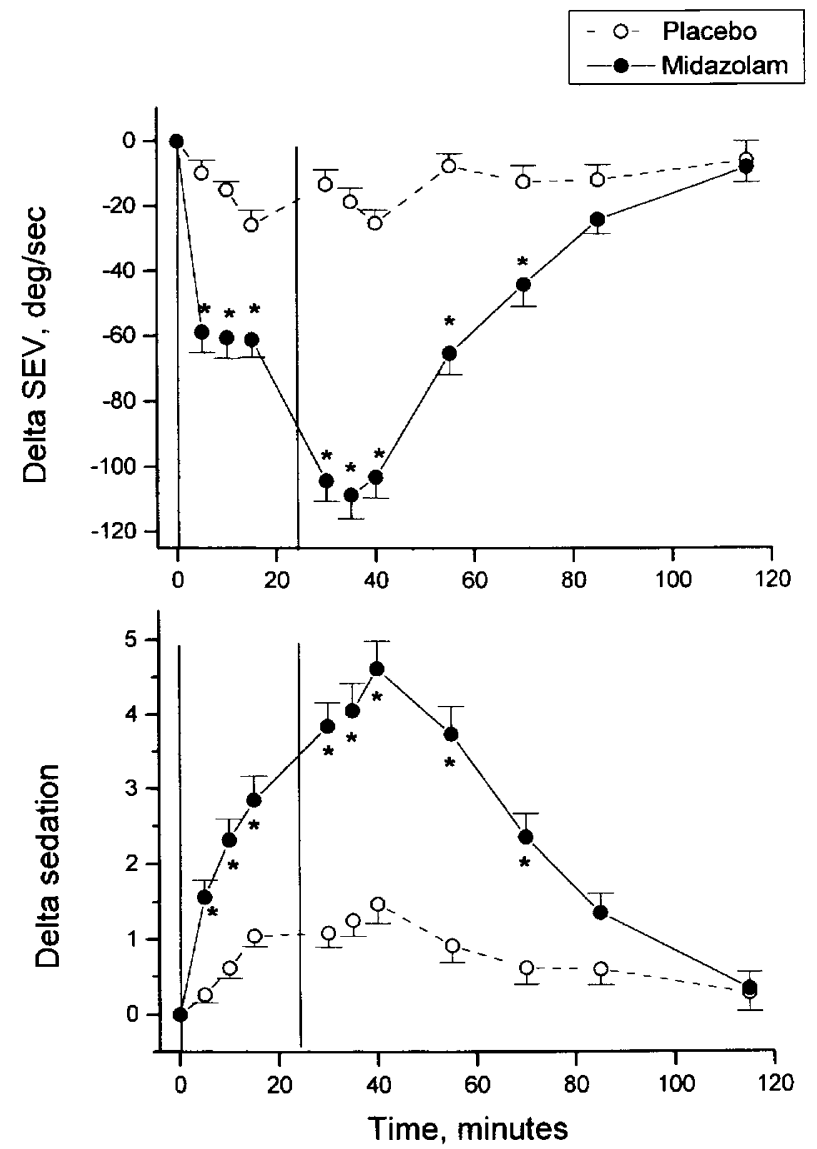

Figure 1. Mean saccadic eye velocity \pm SEM and mean sedation change scores \pm SEM in response to placebo and midazolam. Data from both groups and phases combined. Placebo/midazolam injections indicated by vertical lines. Doses of midazolam were $0.008 \mathrm{mg} / \mathrm{kg}$ and $0.016 \mathrm{mg} / \mathrm{kg}$. There were significant, dose-related decreases in SEV and sedation change scores following midazolam injections $(\mathrm{SEV}: \mathrm{F}(5,499)=2.93 ; p=.013$, sedation: $\mathrm{F}(5,516)=4.13 ; p=$ 001, four-way ANOVA). Post-hoc comparison with Tukey's HSD test. ${ }^{*}$ Difference from placebo, $p<.05$. maximum percent reduction in SEV after midazolam injections was $24.5 \pm 3.5 \%$ and $24.6 \pm 1.7 \%$, respectively. The four-way ANOVA did not reveal any drug by phase effect $\mathrm{F}(1,499)=0,89 ; p=.35$, indicating that the within-group response to midazolam did not change throughout the menstrual cycle. However, when the midazolam response in each group was analyzed separately, the control subjects showed a tendency toward an increased reduction in SEV after midazolam injections in the luteal phase compared to the follicular phase $F(1,114)=3.35 ; p=.07$, whereas the PMS patients did not $\mathrm{F}(1,129)=1.24 ; p=.27$.

\section{Visual Analogue Ratings}

Baseline scores of sedation did not differ between cycle phases in either group (Table 2). Compared to placebo,

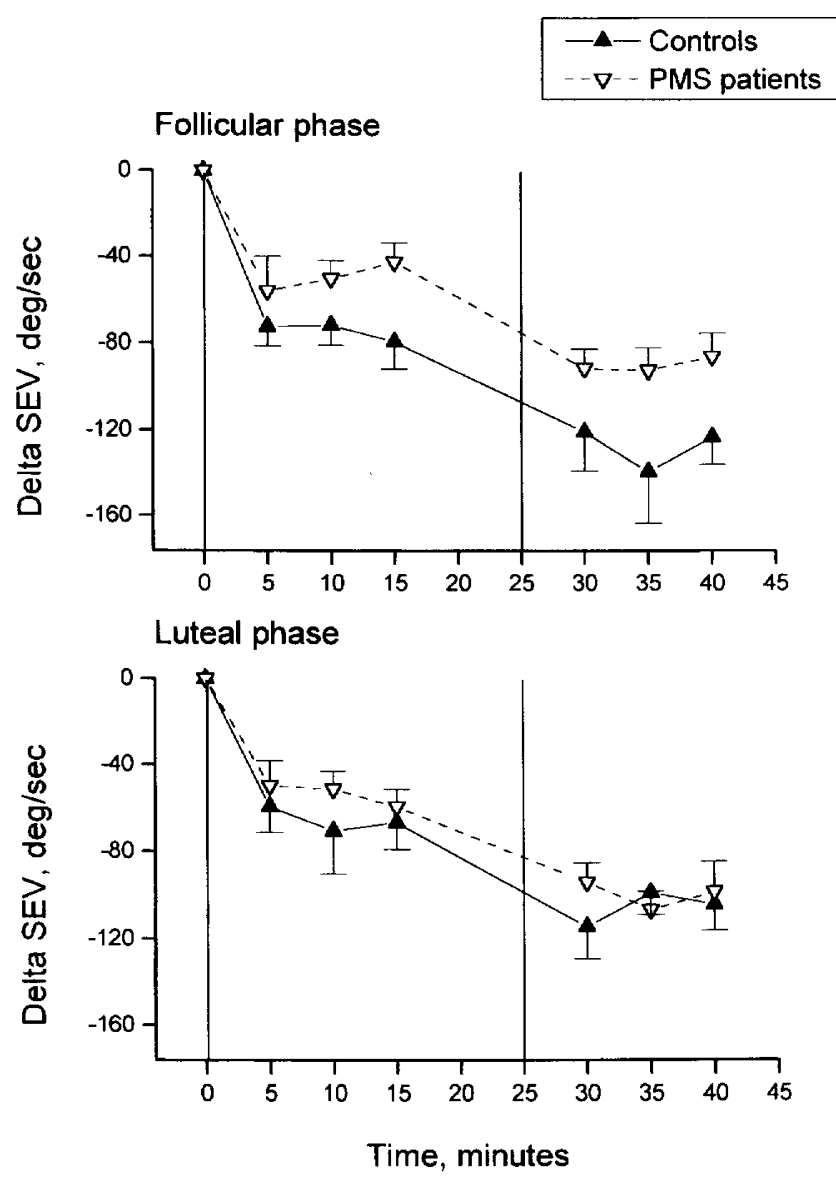

Figure 2. Mean saccadic eye velocity \pm SEM in response to midazolam in the follicular phase (top) and luteal phase (bottom). Midazolam injections indicated by vertical lines. Doses of midazolam were $0.008 \mathrm{mg} / \mathrm{kg}$ and $0.016 \mathrm{mg} / \mathrm{kg}$. PMS patients demonstrated a reduced response to midazolam challenge compared to control subjects in the follicular phase $\mathrm{F}(1,119)=17.73 ; p=.001$ (two-way ANOVA), whereas there was no difference between groups in the luteal phase. 
midazolam injections induced a significant dose-related increase in sedation change scores $\mathrm{F}(5,516)=4.13 ; p=$ .001 (Figure 1). After midazolam injections, control subjects increased their sedation ratings in both cycle phases (follicular phase $\mathrm{F}(5,60)=3.89 ; p=.0041$, luteal phase $F(5,66)=3.41 ; p=.008$, see Figure 3). PMS patients, on the other hand, increased their sedation ratings after midazolam injections only in the follicular phase $F(5,66)=4.64 ; p=.001$, but not in the luteal phase $\mathrm{F}(5,66)=1.97 ; p=.095$ (Figure 3). Placebo injections did not induce any increase in sedation change scores in either group or phase (not shown).

The four-way ANOVA on sedation change scores revealed a significant drug by group interaction where PMS patients responded with less increase in sedation ratings following midazolam compared to control subjects $F(1,516)=10.23 ; p=.001$ (Figure 3 ). When each cy-
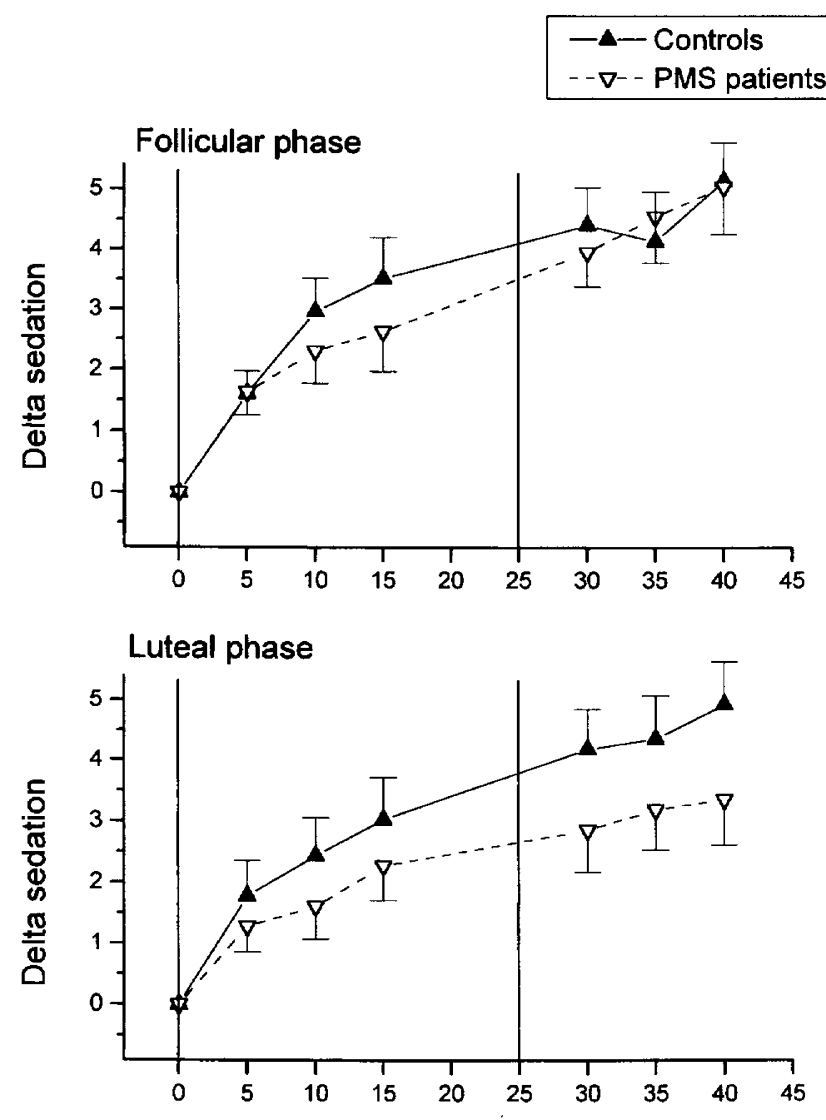

Time, minutes

Figure 3. Mean sedation change scores \pm SEM in response to midazolam in the follicular phase (top) and luteal phase (bottom). Midazolam injections indicated by vertical lines. Doses of midazolam were $0.008 \mathrm{mg} / \mathrm{kg}$ and $0.016 \mathrm{mg} / \mathrm{kg}$. PMS patients demonstrated a reduced response to midazolam in the luteal phase compared to control subjects $\mathrm{F}(1,132)=7.75 ; p=.006$ (two-way ANOVA), whereas there was no difference between groups in the follicular phase. cle phase was analyzed separately by two-way ANOVA on the post-benzodiazepine sedation change scores, this difference between groups in sedation response reached significance in the luteal phase $\mathrm{F}(1,132)=7.75 ; p=.006$, but not in the follicular phase $\mathrm{F}(1,126)=0.57 ; p=.45$. The two-way ANOVA on benzodiazepine sedation response between cycle phases, revealed that PMS patients decreased their change in sedation scores in the luteal phase compared to the follicular phase $F(1,132)=$ $6.73 ; p=.011$. Control subjects did not change their sedation response to benzodiazepines across the menstrual cycle $\mathrm{F}(1,126)=0.22 ; p=.64$. Four PMS patients reached the highest score for sedation prior to the last time-point ( $15 \mathrm{~min}$ after the second midazolam injection) in the follicular phase, whereas in the luteal phase only two PMS patients rated the highest score for sedation on the visual analogue scale. It is therefore unlikely that the group difference in sedation response is due to a ceiling effect in the PMS patients.

No other rated mood symptoms changed significantly throughout the testing days in either group or cycle phase.

\section{Correlations between Saccadic Eye Velocity and Visual Analogue Ratings on Testing Day}

There were significant inverse correlations between sedation change scores and SEV after midazolam injections among the control subjects in both phases (follicular phase: $r=-0.26 ; p=.027$, luteal phase: $r=-0.26$; $p=$ .024). PMS patients showed a significant inverse correlation between sedation change scores and SEV in the follicular phase, $r=-0.34 ; p=.002$, but not in the luteal phase, $r=-0.04 ; p=.72$.

\section{Premenstrual Symptom Severity}

To further evaluate whether the reduced responsiveness to benzodiazepines could be due to a difference in symptom severity, the PMS patients were subdivided into two groups according to the counting of expressed symptoms, described in the methods section. Baseline SEV was not different between the two subgroups $\mathrm{F}(1,40)=0.042 ; p=.84$. The results after midazolam injections are displayed in Figures 4 and 5 . When the three time-points, corresponding to the $8-\mu \mathrm{g} / \mathrm{kg}$ dose of midazolam, were used in the analysis, the four-way ANOVA revealed a significant group (high-severity vs. low-severity PMS patients) by drug interaction $\mathrm{F}(1,120)=$ $28.30 ; p=.001$, indicating that high-severity PMS patients responded with less decrease in SEV after the lower dose of midazolam compared to the low-severity patients. The two-way ANOVA on data from the first midazolam dose showed significant differences between groups in both phases (follicular phase $\mathrm{F}(1,30)=$ $15.16 ; p=.001$, luteal phase $\mathrm{F}(1,30)=7.16 ; p=.012$ ) 
(Figure 4). However, there was no difference between the subgroups of PMS patients after the higher dose of midazolam (Figure 4). The four-way ANOVA on sedation change scores showed a significant group by drug by phase interaction $\mathrm{F}(1,240)=4.85 ; p=.029$ (Figure 5 ). Further analysis by two-way ANOVA on the post-benzodiazepine sedation response confirmed that high-severity PMS patients responded with less increase in sedation ratings after midazolam injections compared to low-severity patients in the luteal phase $\mathrm{F}(1,60)=46.54$; $p=.001$, but not in the follicular phase $\mathrm{F}(1,60)=0.27$; $p=.61$ (Figure 5). Baseline mean sedation scores in the

\section{- $\nabla^{-}$High-symptom PMS} - - Low-symptom PMS

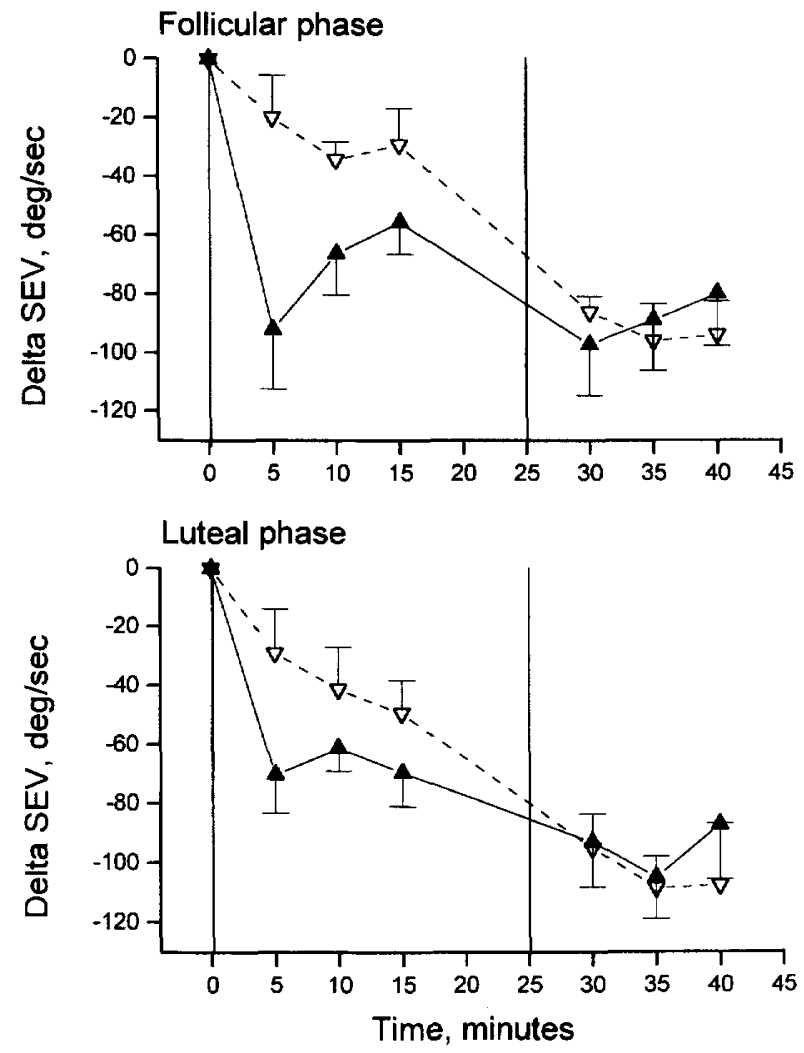

Figure 4. Mean saccadic eye velocity \pm SEM in response to midazolam in the follicular phase (top) and luteal phase (bottom) compared between high-severity PMS patients and low-severity PMS patients. Injections of midazolam indicated by vertical lines. Doses of midazolam were $0.008 \mathrm{mg} /$ $\mathrm{kg}$ and $0.016 \mathrm{mg} / \mathrm{kg}$. The high-severity PMS patients showed a reduced SEV response in both cycle phases after the lowest dose of midazolam compared to low-severity PMS patients (follicular phase $\mathrm{F}(1,30)=15.16 ; p=.001$, luteal phase $\mathrm{F}(1,30)=7.16 ; p=.012$, two-way ANOVA on data corresponding to the lower dose of midazolam). There was no difference between groups after the higher dose of midazolam. luteal phase was $4.78 \pm 1.5$ among the high-severity patients and $3.44 \pm 0.96$ in the low-severity group.

\section{DISCUSSION}

The main finding of the present study was that PMS patients showed a reduced responsiveness to midazolam in comparison with control subjects. Using SEV as dependent measure, the reduced sensitivity to midazolam was most apparent in the follicular phase, whereas the sedation response was reduced in the luteal phase.

PMS patients had lower baseline SEV compared to control subjects. As the ANCOVA analyses preserved the findings of the delta SEV scores, the baseline difference was not considered to be a confounder of the ben-

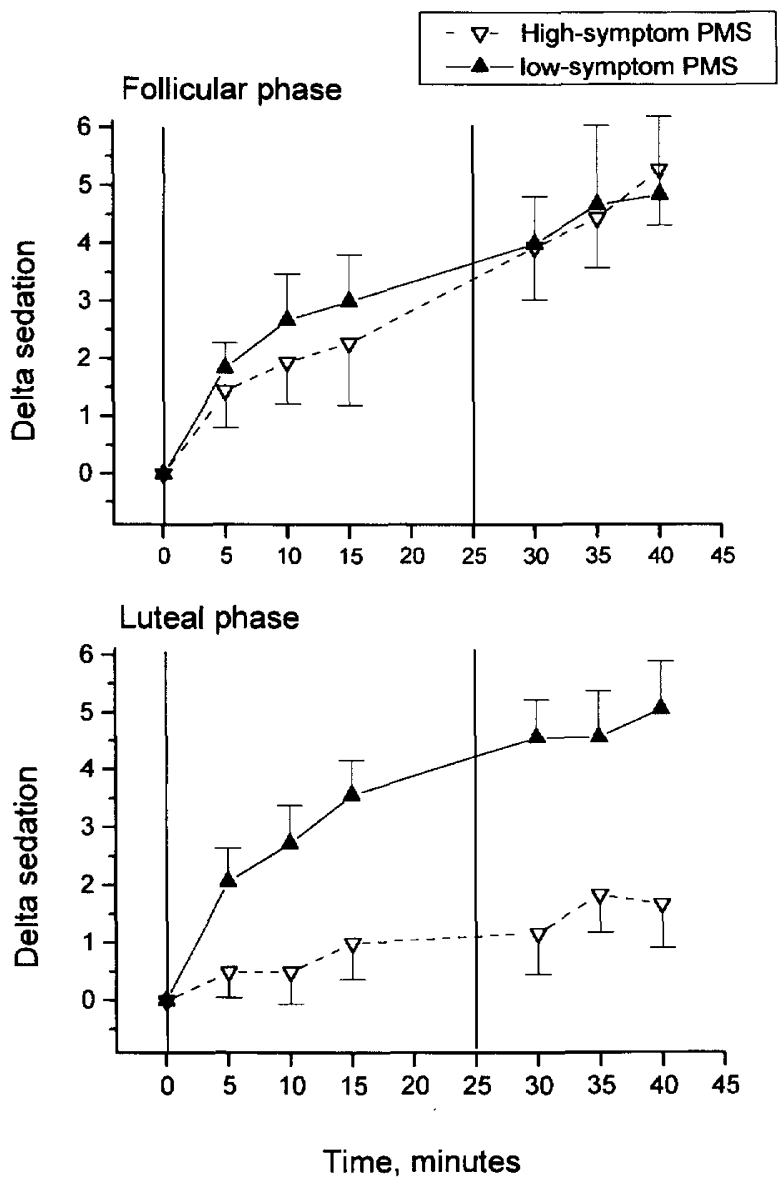

Figure 5. Mean sedation change scores \pm SEM after midazolam injections in the follicular phase (top) and luteal phase (bottom) compared between high-severity PMS patients and low-severity PMS patients. Injections of midazolam indicated by vertical lines. Doses of midazolam were $0.008 \mathrm{mg} /$ $\mathrm{kg}$ and $0.016 \mathrm{mg} / \mathrm{kg}$. In the luteal phase, the high-severity PMS patients responded with less increase in sedation selfratings after midazolam injections compared to low-severity patients $\mathrm{F}(1,60)=46.54 ; p=.001$ (two-way ANOVA). 
zodiazepine results. Furthermore, it has been stated that a 30\% reduction in SEV represents the physiological limitations of the saccadic eye movement system (Cowley et al. 1993). PMS patients in the follicular phase showed a maximum $22 \%$ decrease in SEV and had therefore not reached their floor of performance. Control subjects, on the other hand, showed a 30\% decrease in SEV in the follicular phase, further illustrated by the large number of missing values at this stage of the menstrual cycle. PMS patients often report sleep disturbances in the luteal phase, but possible sleep deprivation among PMS patients could not have accounted for the baseline differences in the follicular phase where patients are asymptomatic. A decreased baseline SEV could also indicate an altered GABAergic tonic inhibition on saccade-related cells of the superior colliculus, but due to the unexpected nature of the finding and the small sample size we believe it should be considered a chance finding until replicated.

As expected, midazolam injections induced significant slowing of SEV and increases in sedation ratings in both groups and cycle phases. There were significant inverse correlations between sedation and SEV in control subjects in both cycle phases and in PMS patients in the follicular phase. These findings are consistent with previous studies on benzodiazepine effects on SEV and sedation (Ball et al. 1991; Hommer et al. 1986).

In comparison to control subjects, PMS patients overall responded with less slowing of SEV after midazolam injections in the follicular phase, less increase in sedation ratings in the luteal phase, and displayed an absent correlation between the SEV and sedation change scores in the luteal phase. These data indicate that PMS patients have a reduced functional sensitivity at the benzodiazepine/GABA $\mathrm{A}_{\mathrm{A}}$-receptor, present in both phases of the menstrual cycle. The different effects of these two measures could be due to the fact that SEV is not merely related to states of arousal, as several studies have been able to distinguish between self-ratings in sedation and slowing of saccadic velocity (Glue et al., 1992; Hopfenbeck et al. 1995). In addition, SEV has been proposed to be a more sensitive measure on benzodiazepine receptor sensitivity compared to sedation ratings (Roy-Burne et al. 1990). The findings of the present study are partly in line with our previous pilot study, where diazepam was used for the benzodiazepine challenge (Sundström et al. 1997). Using SEV and sedation as dependent measures, the pilot study indicated that PMS patients were less sensitive to benzodiazepines compared to controls, especially in the luteal phase. There was a trend toward a reduced sensitivity in the follicular phase as well, a finding which reached significance in the present study where a larger sample was used. However, we were not able to confirm the luteal findings of the pilot study. There are several possible explanations to this; first, the findings of the pilot study may have been mere chance findings. However, since the reduced luteal phase sedation response to benzodiazepines in PMS patients was confirmed by this study, this appears unlikely. Second, the divergent results might be due to the design of the study and/or to the use of a different benzodiazepine compound. Diazepam is a lipid-soluble benzodiazepine with a maximum CNS effect after 1-2 min, whereas the water-soluble midazolam has its maximum CNS effects after 5-10 min (Buhrer et al. 1990). The half-life of diazepam is considerably longer than midazolam ( 33 vs. 2.8 $\mathrm{h}$ ), and the sedative effect of diazepam is more linear than that of midazolam (Reves et al. 1985). It is therefore possible that the divergent results are due to different drug actions. Furthermore, using midazolam in a study of benzodiazepine sensitivity, Ball et al. (1991) reported of a phenomenon suggestive of an acute tolerance mechanism, i.e., a rapid return of SEV toward baseline values within $15 \mathrm{~min}$ after each benzodiazepine injection. This phenomenon was encountered in the present study as well, among the control subjects after the second dose of midazolam in the luteal phase. This rapid decline in benzodiazepine effect on SEV in control subjects might have obscured a possible group difference in the luteal phase. The mechanism of acute tolerance is unclear but the increase with subsequent doses (Ball et al. 1991), and the rapidity in its development may indicate alterations in brain sensitivity rather than metabolic tolerance (Wong et al. 1986). As yet, it is not clear why this phenomenon occurred in control subjects only, but hypothetically it may be due to increased sensitivity to endogenous GABA-positive steroids in the luteal phase. We have conducted a similar challenge, using pregnanolone (a GABA-positive progesterone metabolite), and preliminary data indicate an increased sensitivity to this endogenous compound in the luteal phase among control subjects (Sundström et al. unpublished).

Furthermore, the disadvantages of the EOG, including susceptibility to blink artifacts, poor signal-to-noise ratio, and susceptibility to changes in skin conductance, could also have affected the luteal phase response due to increased arousal among PMS patients at this stage. However, the most likely explanation for the conflicting results between the two studies is that group differences in the luteal phase are less reproducible due to differences in the timing of testings and to differences in PMS symptom severity. Results on biochemical markers on serotonin metabolism and function also demonstrate the difficulty of reproducing luteal phase findings in PMS patients. The blunted prolactin response to tryptophan challenge in the premenstrual phase reported by Bancroft et al. (1991) has not been confirmed (Veeninga and Westenberg 1992). The 5-HT uptake and contents in platelets reported to be significantly reduced in PMS patients in the luteal phase (Ashby et al. 1988) was contradicted by others (Rojansky et al. 1991; 
Veenings and Westenberg 1992). The luteal phase is characterized by rapid changes in plasma levels of estradiol, progesterone, and GRC-active progesterone metabolites (Wang et al. 1996), and the plasma level of progesterone certainly plays a role for the benzodiazepine sensitivity among control subjects. Progesterone treatment has been shown to enhance the sensitivity to triazolam as measured by several psychomotor tests in post-menopausal women (McAuley et al. 1995), and peripheral GABA-levels have been reported to increase in the late luteal phase compared to the mid-follicular phase in control subjects (Halbreich et al. 1996). In our previous study, control subjects increased their benzodiazepine sensitivity in the luteal phase compared to the follicular phase, whereas in the present study, benzodiazepine sensitivity remained unaltered throughout the menstrual cycle among control subjects. It is therefore likely that the timing of measurements, with respect to the progesterone levels in control subjects, plays a role for obtaining optimal conditions for between-group analyses of benzodiazepine sensitivity. Furthermore, the severity of PMS symptoms during the actual study cycle is likely to contribute to the betweengroup differences in the luteal phase. High-severity PMS patients showed a reduced SEV responsiveness to midazolam in both cycle phases, and a reduced sedation response in the luteal phase compared to low-severity patients. These findings are, however, preliminary as the sample size was extremely small. Moreover, the difference between the PMS subgroups in sedative response is likely due to a ceiling effect, operating in the high-severity group. However, although preliminary this finding indicates that the actual severity of PMS symptoms during the study cycle will contribute to the overall difference between PMS patients and control subjects. Another interesting point was the similar phenomenon of acute tolerance which occurred in the lowseverity patients after the first dose of midazolam. This findings may indicate that, during less symptomatic cycles, the midazolam response in PMS patients approaches that of control subjects.

A limitation to the study was the lack of midazolam plasma levels throughout the testings. Previous studies have shown poor correlations between plasma levels and psychomotor effects of benzodiazepines (Linnoila et al. 1983; Ingum et al. 1994), and we were not aware of any dissimilarities in benzodiazepine pharmacokinetics between PMS patients and controls. Similar plasma levels are, however, by no means guaranteed with per $\mathrm{kg}$ dosing and measuring plasma levels of midazolam in the present study would have been helpful in ruling out distributional errors.

The reduced SEV and sedation response to benzodiazepines in PMS patients was evident in both cycle phases. We had predicted, from our previous data, that there would be a marked subsensitivity in the luteal phase. The present study, however, demonstrated that the functional subsensitivity of the benzodiazepine/ $\mathrm{GABA}_{\mathrm{A}}$ receptor complex was apparent throughout the menstrual cycle and is therefore not due to fluctuations in gonadal hormones. This GABAergic subsensitivity rather represents a trait finding that in turn could render the PMS patients susceptible to the hormonal or environmental changes that finally trigger their symptomatology. The inability to confirm previous SEV results in the luteal phase emphasizes the need for rigorous efforts to provide comparable luteal phase conditions when studying biochemical or neurophysiological markers in PMS patients in comparison with control subjects.

\section{ACKNOWLEDGMENTS}

This work was supported by Swedish Medical Research Council (proj 4X-11198) and CoCencys Inc, Irvine, LA. Professor Hans Stenlund is acknowledged for skillful statistical advice. We thank Dr. Erik Lissbrant for revising the text.

\section{REFERENCES}

American Psychiatric Association (1994): Diagnostic and Statistical Manual of Mental Disorders, 4th edition. Washington, DC, U.S. Department of Health and Human Services

Ashby CR, Carr LA, Cool CL, Steptoe MM, Franks DD (1988): Alteration of platelet serotonergic mechanisms and monoamine oxidase activity in premenstrual syndrome. Biol Psychiatry 24:225-233

Bäckström T, Sanders D, Leask R, Davidson D, Warner P, Bancroft J (1983): Mood, sexuality, hormones, and the menstrual cycle. II. Hormone levels and their relationship to the premenstrual syndrome. Psychosom Med 45:503-507

Ball DM, Glue P, Wilson S, Nutt DJ (1991): Pharmacology of saccadic eye movements in man. 1 . Effects of the benzodiazepine receptor ligands midazolam and flumazenil. Psychopharmacology 105:361-367

Baloh RW, Sills AW, Kumley WE, Honrubia V (1975): Quantitative measurement of saccade amplitude, duration, and velocity. Neurology 25:1065-1070

Bancroft J, Cook A, Davidson D, Bennie J, Goodwin G (1991): Blunting of neuroendocrine responses to infusion of L-tryptophan in women with perimenstrual mood change. Psychol Med 21:305-312

Becker W (1989): Metrics. In Wurtz RH, Goldberg ME (eds), The Neurobiology of Saccadic Eye Movements. Amsterdam, Elsevier Science Publishers BV, pp 13-67

Bitran D, Purdy RH, Kellogg CK (1993): Anxiolytic effects of progesterone is associated with increases in cortical allopregnanolone and $\mathrm{GABA}_{\mathrm{A}}$ receptor function. Pharmacol Biochem Behav 45:423-428

Buhrer M, Maitre PO, Crevoisier C, Stanski DR (1990): Electroencephalographic effects of benzodiazepines. II. Pharmacodynamic modeling of the electroencephalo- 
graphic effects of midazolam and diazepam. Clin Pharmacol Ther 48:555-567

Cowley DS, Roy-Burne PP, Greenblatt DI, Hommer DW (1993): Personality and benzodiazepine sensitivity in anxious patients and control subjects. Psychiatry Res $47: 151-162$

Freeman EW, Rickels K, Sondheimer SJ, Polansky M (1995): A double-blind trial of oral progesterone, alprazolam and placebo in treatment of severe premenstrual syndrome. JAMA 274:51-57

Glue P, Bailey J, Wilson S, Hudson A, Nutt DJ (1992): Thyrotropin-releasing hormone selectively reverses lorazepaminduced sedation but not slowing of saccadic eye movements. Life Sci 50:25-30

Greenblatt DJ, Ehrenberg BL, Gunderman J, Locniskar A, Scavone JM, Harmatz JS, Shader RI (1989): Pharmacokinetic and electroencephalographic study of intravenous diazepam, midazolam, and placebo. Clin Pharmacol Ther 45:356-365

Halbreich U, Petty F, Yonkers K, Kramer GL, Rush AJ, Bibi KW (1996): Low plasma gamma-aminobutyric acid levels during the late luteal phase of women with premenstrual dysphoric disorder. Am J Psychiatry 153:718-720

Hammarbäck S, Ekholm UB, Bäckström T (1991): Spontaneous anovulation causing disappearance of cyclical symptoms in women with the premenstrual syndrome. Acta Endocrinol (Copenh) 125:132-137

Hammabäck S, Bäckström T (1988): Induced anovulation as treatment of premenstrual tension syndrome. Acta Obstet Gynecol Scand 67:159-166

Hammarbäck S, Bäckström T, MacGibbon-Taylor B (1989a): Diagnosis of premenstrual tension syndrome: Description and evaluation of a procedure for diagnosis and differential diagnosis. J Psychosom Obstet Gynaecol $10: 25-42$

Hammarbäck S, Damber J-E, Bäckström T (1989b): Relationship between symptom severity and hormone changes in women with premenstrual syndrome. J Clin Endocrinol Metab 68:125-130

Hikosaka O, Wurtz RH (1985a): Modification of saccadic eye movements by GABA-related substances. I. Effect of muscimol and bicuculline in the monkey superior colliculus. J Neurophysiol 53:266-291

Hikosaka O, Wurtz RH (1985b): Modification of saccadic eye movements by GABA-related substances. II. Effects of muscimol and bicuculline in the monkey substantia nigra pars reticulata. J Neurophysiol 53:292-308

Hommer DW, Matsuo V, Wolkowitz O, Chrousos G, Greenblatt DJ, Weingarter H, Paul SM (1986): Benzodiazepine sensitivity in normal human subjects. Arch Gen Psychiatry $43: 542-551$

Hopfenbeck JR, Cowley DS, Radant A, Greenblatt DJ, RoyBurne PP (1995): Effects of diphenhydramine on human eye movements. Psychopharmacology 118:280-286

Ingum J, Pettersen G, Sager G, Morland J (1994): Relationship between unbound plasma concentrations and various psychomotor and subjective effects after intake of diazepam and flunitrazepam. Int Clin Psychopharmacol 9:115-121

Majewska MD, Harrison NL, Schwartz RD, Barker JL, Paul
SM (1986): Steroid hormone metabolites are barbituratelike modulators of the GABA receptor. Science 232: 1004-1007

Marshall RW, Griffiths AN, Richens A (1985): A microcomputer system to assess CNS depression from the analysis of the dynamics of saccadic eye movements. Br J Clin Pharmacol 20:304-305

Marshall RW, Richens A (1989): An IBM-based system for the generation, collection and analysis of saccadic and smooth pursuit eye movements. Br J Clin Pharmacol 28:752-753

McAuley JW, Reynolds IJ, Kroboth FJ, Smith RB, Kroboth PD (1995): Orally administered progesterone enhances sensitivity to triazolam in postmenopausal women. J Clin Psychopharmacol 15:3-11

McCormack HM, Horne DJdL, Sheather S (1988): Clinical application of visual analogue scales: A critical review. Psychol Med 18:1007-1019

Mercer AJ, Marshall RW, Richens A (1990): Saccadic eye movements as a method of psychometric assessment. In Klepper ID, Saunder LD, Rosen M (eds), Ambulatory Anaesthesia and Sedation. Oxford, Blackwell, pp 88-105

Linnoila M, Erwin CW, Brendle A, Simpson D (1983): Psychomotor effects of diazepam in anxious patients and healthy volunteers. J Clin Psychopharmacol 3:88-96

Okada Y (1992): The distribution and function of $\gamma$-aminobutyric acid (GABA) in the superior colliculus. In Mize RR, Marc RE, Silito AM (eds), Progress in Brain Research. Amsterdam, Elsevier Science Publishers BV, pp 249-262

Parry BL, Gerner RH, Wilkins JN, Halaris AE, Carlson HE, Hershman JM, Linnoila M, Merrill J, Gold PW, Gracely $R$ (1991): CSF and endocrine studies of premenstrual syndrome. Neuropsychopharmacology 5:127-137

Paul SM, Purdy RH (1992): Neuroactive steroids. FASEB J 6:2311-2322

Purdy RH, Morrow AL, Moore PH, Paul SM (1991): Stressinduced elevations of the $\gamma$-aminobutyric acid type $A$ receptor-active steroids in the rat brain. Proc Natl Acad Sci USA 88:4553-4557

Reves JG, Fragen RJ, Vinik HR, Greenblatt DJ (1985): Midazolam: Pharmacology and uses. Anesthesiology 62:310324

Rojansky N, Halbreich U, Zander K, Barkai A, Goldstein S (1991): Imipramine receptor binding and serotonin uptake in platelets of women with premenstrual changes. Gynecol Obstet Invest 31:146-152

Roy-Burne PP, Cowley DS, Greenblatt DJ, Shader RI, Hommer D (1990): Reduced benzodiazepine sensitivity in panic disorder. Arch Gen Psychiatry 47:534-538

Schmidt PJ, Purdy RH, Moore PH, Paul SM, Rubinow DR (1994): Circulating levels of anxiolytic steroids in the luteal phase in women with premenstrual syndrome and in control subjects. J Clin Endocrinol Metab 79:1256-1260

Smith S, Rinehart JS, Ruddock VE, Schiff I (1987): Treatment of premenstrual syndrome with alprazolam: Results of a double-blind, placebo-controlled, randomized crossover clinical trial. Obstet Gynecol 70:37-43

Sundblad C, Modigh K, Andersch B, Eriksson E (1992): Clo- 
mipramine effectively reduces premenstrual irritability and dysphoria: A placebo-controlled trial. Acta Psychiatr Scand 85:39-47

Sundström I, Ashbrook D, Bäckström T (1997): Reduced benzodiazepine sensitivity in patients with premenstrual syndrome, a pilot study. Psychoneuroendocrinology 22:25-38

Wang MD, Seippel L, Purdy RH, Bäckström T (1996): Relationship between symptom severity and steroid variation in women with premenstrual syndrome: Study on serum pregnenolone, pregnenolone sulfate, $5 \alpha-$ preg- nan-3,20-dione and 3 3 -hydroxy-5 $\alpha$-pregnan-20-one. J Clin Endocrinol Metab 81:1076-1082

Veeninga AT, Westenberg HGM (1992): Serotonergic function and late luteal phase dysphoric disorder. Psychopharmacology 108:153-158

Wieland S, Lan NC, Mirasedeghi S, Gee KW (1991): Anxiolytic activity of the progesterone metabolite $5 \alpha$-pregnan-3 $\alpha$-ol-20-one. Brain Res 565:263-268

Wong PT, Yoong YL, Gwee MC (1986): Acute tolerance to diazepam induced by benzodiazepines. Clin Exp Pharmacol Physiol 13:1-8 